\title{
GAMMA GLOBULIN AND COMPLEMENT IN THE DISEASED KIDNEY
}

\author{
By P. FREEDMAN AND A. S. MARKOWITZ \\ (From St. George's Hospital, London, England, and the Hektoen Institute for Medical Research, \\ Chicago, Ill.)
}

(Submitted for publication August 28, 1961 ; accepted September 21, 1961)

Recent studies on the kidney, utilizing the fluorescent antibody technique, have revealed the presence of $\gamma$-globulin in the renal lesions in glomerulonephritis, and in the renal lesions associated with disseminated lupus erythematosus, progressive systemic sclerosis, polyarteritis nodosa, amyloidosis, and diabetes mellitus $(1,2)$. The $\gamma$-globulin has been dissociated from combination with the tissue, and in the experiments with glomeruli isolated from lupus nephritis, the $\gamma$-globulin was found to possess antibody-like activity under certain in vitro conditions (3). The present study was undertaken to determine whether there was evidence for the presence of complement together with $\gamma$-globulin in a small series including normal kidneys, glomerulonephritis, lupus nephritis, and diabetic nephropathy. A preliminary report of these observations has been made (4).

\section{MATERIALS AND METHODS}

\section{Production of antiserum against human complement}

Based upon the method described by McKee and Jeter (5), antiserum was prepared against human complement, utilizing different antigenic moieties for forming the complex, before administration to rabbits. In each instance, freshly drawn human serum served as the source of the complement. All precipitates were washed in the standard manner and combined with complete Freund's adjuvant for intramuscular injection into adult female rabbits.

The antigenic moieties employed were human $\gamma$-globulin, human albumin, bovine $\gamma$-globulin and ova-albumin. The complexes were injected at weekly intervals with the exception of the last, which was injected 16 days after the previous one. The animals were exsanguinated 12 days after the last injection by cardiac puncture.

\section{Anticomplement assay}

The antibody titer against human complement was determined on the basis of previously described studies (5). The anticomplement titer had a final value of $1: 128$.

\section{Specificity of anticomplement serum}

The serum was found to contain an antibody response against human $\gamma$-globulin which was removed by absorption with a slight excess of the antigen. Such a procedure had no detectable effect on the anticomplement titer. In order to exclude a possible antibody response against other human serum proteins (which may not have been completely removed from the antigen-antibody complex during washing), the serum was further absorbed with human serum albumin and a composite fraction which contained human $\alpha_{1},, \alpha_{2}-$ and $\beta$-globulins. Absorption with lyophilized pooled fresh human serum (in order to avoid a dilution factor) reduced the anticomplement titer to zero.

Fresh human serum was decomplemented both by a human antigen-antibody system (human serum albumin and rabbit anti-HSA) and a nonhuman system (bovine $\boldsymbol{\gamma}$-globulin and rabbit anti-BGG). The decomplemented serum in both instances contained no detectable complement as measured in a hemolytic system.

Precipitin ring tests were utilized in an effort to evaluate the specificity of the anticomplement serum. The rabbit antiserum, absorbed with human serum protein $\left(\gamma-, \alpha_{1}-, \alpha_{2}-, \beta\right.$-globulins, and albumin), precipitated with fresh human serum. After absorption of the antiserum with lyophilized pooled fresh human serum, the precipitin reaction against fresh human serum was eliminated. Also, immunologically decomplemented human serum, using both methods described above, failed to precipitate with the rabbit antiserum.

\section{Fluorescent antibody studies}

A portion of the rabbit antiserum was conjugated with fluorescein isothiocyanate (6). The antibody titer of the conjugated serum was reduced to $1: 64$. The conjugated antiserum was used directly on frozen sections as described previously (1) for the direct procedure. The indirect method was also employed, using unconjugated antiserum and fluorescein-conjugated sheep antirabbit globulin. Nonspecific fluorescence was removed as described previously (1).

\section{Specificity of fluorescence}

Direct staining. The fluorescein-conjugated antihuman complement serum did not produce fluorescence on normal kidney tissue. When fluorescence was observed in sections of diseased kidney, prior blocking of the tissue with the unconjugated serum considerably diminished 
the subsequent fluorescence. Absorption of the fluorescent substance with preparations of human $\gamma$-globulin and protein fractions which contained albumin, $\alpha_{1}^{-}, \alpha_{2^{-}}$and $\beta$-globulins did not diminish the subsequent fluorescence. Prior blocking of the sections with rabbit antihuman $\gamma$-globulin did not diminish the subsequent fluorescence. Absorption of the fluorescent substance with lyophilized fresh pooled normal human serum abolished the fluorescence.

Indirect staining. In this procedure unconjugated rabbit antihuman complement serum was allowed to react with the tissue, and after thorough washing the sections were stained with a fluorescein-conjugated sheep antirabbit globulin.

Specificity tests included all those described above for the direct procedure. In addition, blocking with unconjugated sheep antirabbit globulin, after the rabbit anticomplement serum had reacted with the tissue, considerably diminished the fluorescence observed after application of the sheep antirabbit globulin conjugate. Also, the fluorescein-conjugated sheep antirabbit globulin produced no fluorescence after normal rabbit serum had been applied to kidney sections.

\section{RESULTS}

Kidney tissue from 22 patients was examined, by the fluorescent antibody technique, for the presence of complement and also for $\gamma$-globulin. In 16 instances tissue was obtained by biopsy (7) and in 6 cases at autopsy performed shortly after death. Details of the technique employed have been described previously (1). In 6 instances there was no evidence of renal disease, and no specific fluorescence was observed either for $\gamma$-globulin or complement in the kidney sections.

Kidney tissue was also obtained from a patient with scleroderma. Histological sections showed no vascular changes of scleroderma, and the glomeruli, apart from doubtful minimal thickening of the glomerular basement membrane in certain areas, were normal. Fluorescence for $\gamma$-globulin and complement was also negative.

Renal biopsy was also undertaken in a young woman who had developed acute glomerulonephritis 6 months previously. The first biopsy had shown an acute proliferative glomerulonephritis with exudation, and immunological studies revealed $\gamma$-globulin deposition in the glomerular basement membrane. During the interim period there had been steady improvement in her clinical condition and at the time of the second biopsy she was well, symptom-free, and the urine was normal. The second biopsy showed some residual glomerular hypercellularity but no basement membrane changes. The kidney tissue was negative for $\gamma$-globulin and complement.

Specific fluorescence, indicative of $\gamma$-globulin and also of complement, was found in kidney tissue from four patients with various forms of glomerulonephritis (Figures 1 and 2) and from seven patients with renal involvement in disseminated lupus erythematosus (Figures 3 and 4). Fluorescence was observed in the glomerular capillary walls in all cases, and in some of the tubules and blood vessel walls in approximately half the sections studied. Renal biopsy from a woman aged 42 , recently discovered on routine urine testing to have diabetes mellitus, was also found to contain well marked specific fluorescence for both $\gamma$-globulin and complement, in the glomeruli (Figures 5 and 6) and also in the tubular basement membrane area and the walls of small blood vessels.

Kidney tissue, obtained by biopsy, was examined from a young girl with evidence of HenochSchönlein purpura with renal involvement. Histological examination revealed mild focal proliferative and membranous changes in the glomeruli. Fluorescent antibody studies showed weak specific fluorescence for both $\gamma$-globulin and complement in the glomerular capillary walls. The main features of these cases are outlined in Table I. In general, the direct and indirect techniques gave closely comparable results, the latter technique probably yielding slightly greater sensitivity.

Throughout the sections examined, the presence of complement in the glomeruli was always associated with $\gamma$-globulin, and the pattern of distribution within the glomerulus was similar. Care was always taken to ensure that no cross reaction occurred between the anticomplement serum and $\gamma$-globulin, and the control tests described above were frequently applied. With regard to specific fluorescence in the tubules, usually observed in the basement membrane area and in the blood vessel walls, there were instances where either $\gamma$-globulin, or more usually, complement was found, without the apparent presence of the other. In this connection it may be pertinent to observe that fluorescence in these situations was neither so intense nor so uniform as in the glomeruli. With small pieces of tissue these changes were observed less easily, and where, as in the present investigation, larger numbers of sections from a biopsy were devoted to staining with the anti- 

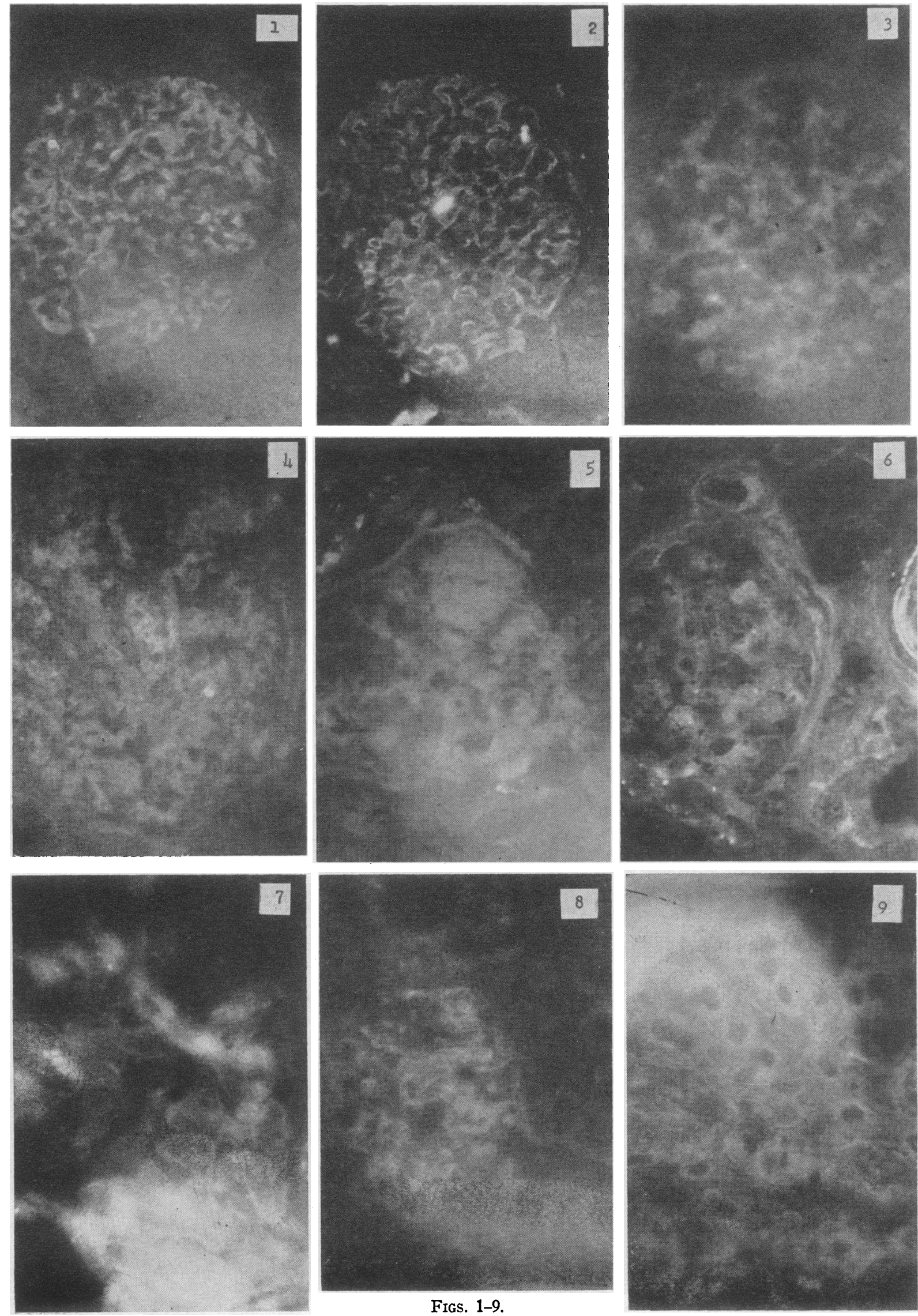

FIGS. 1-9. 
complement fluorescent substance, some apparent discrepancy in the localization patterns of complement and $\gamma$-globulin might occur.

In the case of kidney sections from patients with disseminated lupus erythematosus, reference has previously been made to the patchy nature of the specific fluorescence for $\gamma$-globulin within the glomerular tuft (1), and a similar localization of the specific fluorescence for complement was observed. Examination of many preparations and the use of higher magnification suggested a nuclear localization of specific fluorescence in the glomeruli in several instances, both for $\gamma$-globulin and for complement. This sometimes appeared as fluorescence, either in nuclei or nuclear fragments, or as specific fluorescence in or around the nuclear membrane (Figures 7, 8 and 9). One section of kidney tissue from a patient with lupus erythematosus was stained with the anticomplement fluorescent substance, and well marked nuclear fluorescence was seen in cells within the lumen of a moderate sized vessel; fluorescence was also seen in the tubular nuclei in some areas. This pattern of fluorescence was not observed in glomerulonephritis or in diabetic nephropathy.

The seven patients with lupus nephritis had received varying treatment with steroids before they were studied: two had received prolonged steroid therapy, three had been given short courses, and two had had no steroid therapy. In this small group of cases the administration of steroids did not appear to influence the localization of either $\gamma$-globulin or complement in the kidney. The serum of five of these seven patients was examined for the presence of a circulating antinuclear factor $(2,8)$, and this was found in all five.

Fig. 1. Glomerulonephritic Kidney Stained For $\gamma$-GLobulin. Frozen section of kidney from patient with mild membranous and proliferative glomerulonephritis (Patient 1 ), stained with fluorescein-conjugated horse antihuman $\gamma$-globulin $(\times 560)$. Fluorescence seen through ultraviolet light microscope, indicative of $\gamma$-globulin, present in glomerular capillary wall.

Fig. 2. Glomerulonephritic Kidney stained for Complement. Kidney section from same patient as in Figure 1, first treated with unconjugated rabbit antihuman $\gamma$-globulin, and then stained with rabbit antihuman complement conjugated with fluorescein isothiocyanate, previously absorbed with human $\alpha_{1^{-}}, \alpha_{2}-, \beta$-, and $\gamma$-globulins and albumin $(\times 560)$. Fluorescence again visible in glomerular capillary wall.

Fig. 3. Lupus erythematosus Kidney Stained for $\boldsymbol{\gamma}$-GLobulin. Glomerulus from patient with systemic lupus erythematosus (Patient 8 ) stained with fluorescein-conjugated horse antihuman $\gamma$-globulin $(\times 560)$. Patchy fluorescence visible in glomerular capillary wall.

Fig. 4. Lupus erythematosus kidney stained for complement. Glomerulus from same patient as in Figure 3, first treated with unconjugated rabbit antihuman complement, previously absorbed with human $\gamma$-globulin, and then stained with fluorescein-conjugated sheep antirabbit $\gamma$-globulin $(\times 560)$. Patchy fluorescence in glomerular capillary wall.

Fig. 5. Diabetic Kidney StAined For $\gamma$-GLOBulin. Glomerulus from patient with diabetic nephropathy (Patient 5), stained with fluorescein-conjugated horse antihuman $\gamma$-globulin $(\times 560)$. Fluorescence visible in glomerular capillary wall, glomerular nodules, and in wall of small blood vessel adjacent to glomerulus.

Fig. 6. Diabetic KidNey StAined for complement. Glomerulus from same patient as in Figure 5, first treated with rabbit antihuman $\gamma$-globulin, then with rabbit antihuman complement previously absorbed with human $\gamma$-globulin, and stained with fluorescein-conjugated sheep antirabbit $\gamma$-globulin $(\times 560)$. Fluorescence seen in glomerular capillary wall and nodules and in wall of small blood vessel adjacent to glomerulus.

Fig. 7. HIGH-POWER VIEW OF SPECIFIC FLUORESCENCE FOR $\gamma$-GLOBULIN IN LUPUS ERYTHEMAToSUS GLOMERULUs. Portion of glomerulus from patient with systemic lupus erythematosus (Patient 8) stained with fluorescein-conjugated horse antihuman $\gamma$-globulin $(\times 1280)$. Fluorescence appears concentrated, in areas, around nuclei.

Fig. 8. LupUs eRythematosus Kidney StAined For Complement. Glomerulus from patient with systemic lupus erythematosus (Patient 6), first treated with rabbit antihuman complement previously absorbed with human $\gamma$-globulin, and then stained with fluorescein-conjugated sheep antirabbit $\gamma$-globulin $(\times 560)$. Fluorescence visible in some nuclei, as well as in glomerular capillary wall.

Fig. 9. High-power view of Specific fluorescence for complement in lupus erythematosus glomerulus. Portion of glomerulus from same patient as in Figure 8, and stained in same manner for human complement $(\times 1280)$. Fluorescence visible around nuclei. 


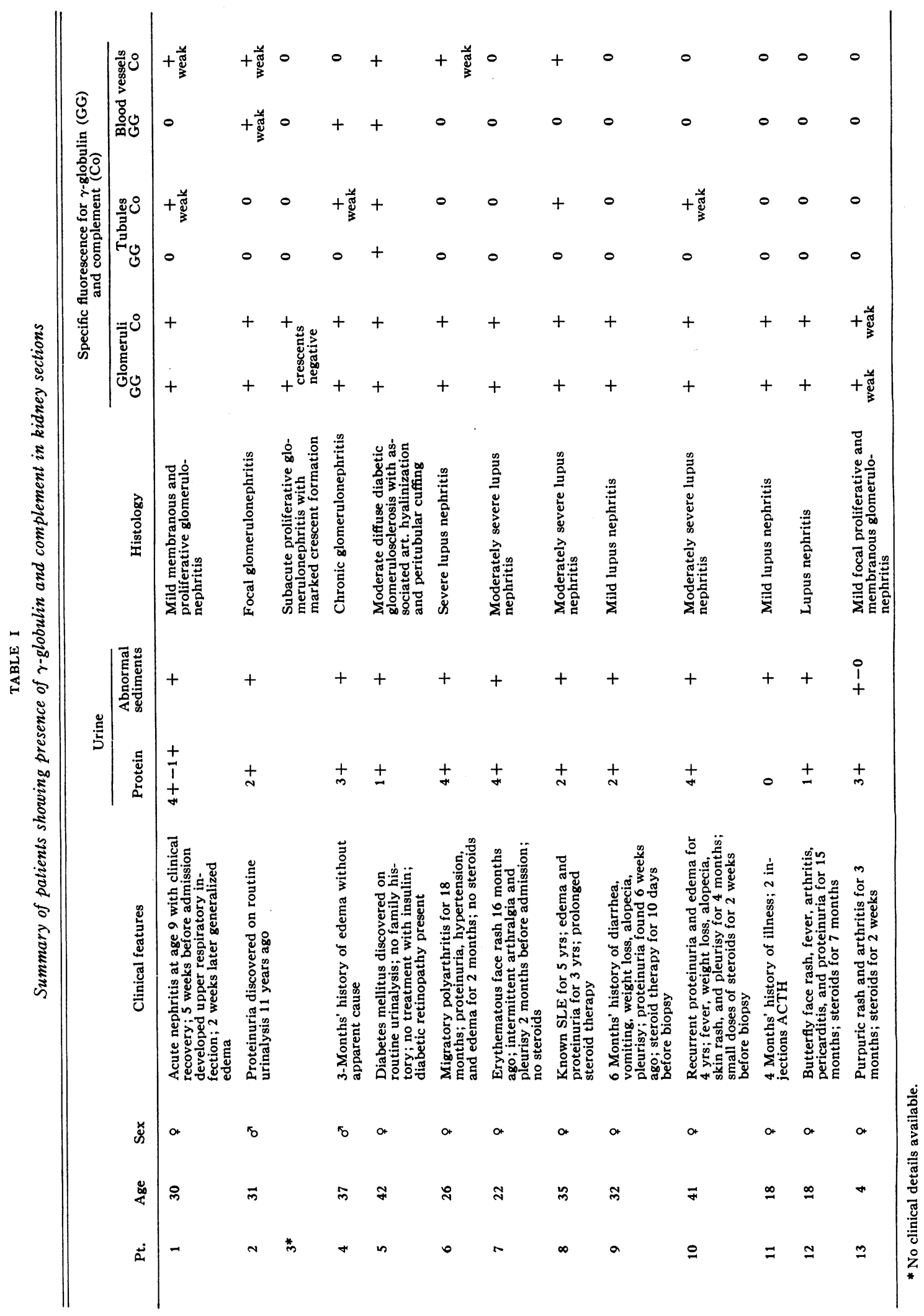




\section{DISCUSSION}

A considerable volume of experimental data has accrued since the initial production of nephrotoxic nephritis by Lindemann (9), and this has been reviewed recently (10). The evidence, drawn from many sources, has indicated that an antigen-antibody reaction is involved in the kidney lesions in glomerulonephritis and in the renal lesions associated with the connective tissue diseases. Mellors, Ortega and Holman applied the fluorescent antibody technique to sections of human kidney obtained at autopsy and found $\gamma$-globulin in the renal lesions in glomerulonephritis, polyarteritis nodosa, and renal amyloidosis $(11,12)$. These studies were later extended to larger series with mainly confirmatory results (1, $2,13)$. Since the presence of $\gamma$-globulin did not in itself constitute evidence of antibody, further studies were undertaken with a view to defining the role of this globulin. Elution procedures conducted at $\mathrm{pH} 3.2$ to 3.5 , a medium known to favor the dissociation of many antigen-antibody complexes, permitted the release of $\gamma$-globulin from frozen kidney sections in glomerulonephritis, polyarteritis nodosa, and lupus nephritis, as well as in one case of diabetic nephropathy studied in the same way (1). Subsequent studies on isolated glomeruli from a normal kidney and from kidneys of patients with glomerulonephritis and lupus nephritis confirmed these elution studies (3). Also, the $\gamma$-globulin eluted from the kidney of the patient with lupus nephritis, in the presence of a heat-labile factor in normal serum, was shown to possess antinuclear activity, and therefore to behave as an antibody.

The present study was concerned with the localization of complement, and this has provided evidence that an antigenic complex, reacting immunologically as complement, is present in the diseased kidney together with $\gamma$-globulin. This would appear to provide further evidence of the antibody nature of the $\gamma$-globulin localized in the renal lesions in the diseases discussed above.

The apparent nuclear localization of $\gamma$-globulin and complement in some sections in lupus nephritis is of interest in the light of the known presence of circulating antinuclear antibody in this disease (8). If the results in this small series are representative of this disease group as a whole, the role of the antinuclear antibody in the pathogenesis of the renal lesions may assume greater significance. However, it may still be argued that the nuclear changes observed are subsequent to some more basic primary tissue reaction. In any event it would appear that the antinuclear antibody may be concerned in the development of the renal lesions, whether in a primary or secondary role.

Final proof of the presence of complement in these situations requires the presence of a pure preparation of human complement. In the absence of this, interpretation of these results must remain inferential.

Shortly after these observations had been described in a preliminary communication (4), Klein and Burkholder reported independently the localization of complement in the glomeruli in nephrotoxic nephritis in the rat (14).

\section{SUM MARY}

The production of an antiserum to human complement, in rabbits, is described. This antiserum and antisera to human $\gamma$-globulin were used to study sections of human kidney by the fluorescent antibody technique.

Neither $\gamma$-globulin nor complement was found in normal kidneys, in the kidney of a patient who had recovered from acute glomerulonephritis, or in the kidney of a patient with scleroderma with doubtful histological changes. Both substances were present, in a closely comparable distribution, in the renal lesions in glomerulonephritis and lupus nephritis, and in one case of diabetic nephropathy.

The glomerular localization of the specific fluorescence in lupus nephritis is discussed in relation to the circulating antinuclear antibody present in this disease.

\section{ACKNOWLEDGMENTS}

Part of this work was undertaken while one of the authors (P.F.) was the Bilton Pollard Fellow of University College Hospital Medical School, at The Department of Medicine, Research and Educational Hospital, University of Illinois, in the department of Professor R. M. Kark, whose help we gratefully acknowledge. We should also like to thank Professor M. L. Rosenheim and Professor A. C. Dornhorst for their valuable help, encouragement, and criticism, and $\mathrm{Mr}$. 
Anatoly Bezcorovainy, M.S., Department of Biochemistry, University of Illinois College of Medicine, for supplying the purified human serum protein fractions.

\section{REFERENCES}

1. Freedman, P., Peters, J. H., and Kark, R. M. Localization of gamma-globulin in the diseased kidney. A.M.A. Arch. intern. Med. 1960, 105, 524.

2. Freedman, P. Lupus nephritis in Recent Advances in Renal Disease, M. D. Milne, Ed. London, Pitman Medical Publ. Co., 1960, p. 90.

3. Freedman, P., and Markowitz, A. S. Isolation of antibody-like gamma globulin from lupus glomeruli. In press.

4. Freedman, P., and Markowitz, A. S. Immunological studies in nephritis. Lancet 1959, 2, 45.

5. McKee, A. P., and Jeter, W. S. The demonstration of an antibody against complement. J. Immunol. 1956, 76, 112.

6. Marshall, J. D., Eveland, W. C., and Smith, C. W. Superiority of fluorescein isothiocyanate (Riggs) for fluorescent-antibody technic with a modification of its application. Proc. Soc. exp. Biol. (N. Y.) 1958, 98, 898.

7. Kark, R. M., and Muehrcke, R. C. Biopsy of kidney in prone position. Lancet 1954, 1, 1047.

8. Friou, G. J., Finch, S. C., and Detre, K. D. Interaction of nuclei and globulin from lupus erythema- tosus serum demonstrated with fluorescent antibody. J. Immunol. 1958, 80, 324.

9. Lindemann, W. Sur le mode d'action de certains poisons rénaux. Ann. Inst. Pasteur 1900, 14, 49.

10. Peters, J. H., and Freedman, P. Immunologic aspects of renal disease. New Engl. J. Med. 1959, 261, 1166.

11. Mellors, R. C., and Ortega, L. G. Analytical pathology. III. New observations on pathogenesis of glomerulonephritis, lipid nephrosis, periarteritis nodosa, and secondary amyloidosis in man. Amer. J. Path. 1956, 32, 455.

12. Mellors, R. C., Ortega, L. G., and Holman, H. R. Role of gamma globulins in pathogenesis of renal lesions in systemic lupus erythematosus and chronic membranous glomerulonephritis, with observation on lupus erythematosus cell reaction. J. exp. Med. 1957, 106, 191.

13. Taft, L. I., Dineen, J. K., and MacKay, I. R. Localization and binding of serum proteins in glomeruli of kidney biopsies in disseminated lupus erythematosus and glomerulonephritis. Aust. Ann. Med. 1958, 7, 5.

14. Klein, P., and Burkholder, P. Ein Verfahren zur fluoreszenzoptischen Darstellung der Komplementbindung und seine Anwendung zur histo-immunologischen Untersuchung der experimentellen Nierenanaphylaxie. Dtsch. med. Wschr. 1959, 84, 2001. 